\title{
WEAK CONVERGENCE OF AN ITERATIVE SCHEME FOR GENERALIZED EQUILIBRIUM PROBLEMS
}

\author{
JIAN-WEN PENG ${ }^{\bowtie}$ and JEN-CHIH YAO
}

(Received 3 August 2008)

\begin{abstract}
In this paper, we introduce an iterative scheme using an extragradient method for finding a common element of the set of solutions of a generalized equilibrium problem, the set of fixed points of a nonexpansive mapping and the set of the variational inequality for a monotone, Lipschitz-continuous mapping. We obtain a weak convergence theorem for three sequences generated by this process. Based on this result, we also obtain several interesting results. The results in this paper generalize and extend some well-known weak convergence theorems in the literature.
\end{abstract}

2000 Mathematics subject classification: primary 47H10, 54H25.

Keywords and phrases: generalized equilibrium problem, extragradient method, nonexpansive mapping, monotone mapping, variational inequality, weak convergence, fixed point.

\section{Introduction}

Let $H$ be a real Hilbert space with inner product $\langle\cdot, \cdot\rangle$ and induced norm $\|\cdot\|$. Let $C$ be a nonempty closed convex subset of $H$. Let $F$ be a bifunction from $C \times C$ to $R$ and let $B: C \rightarrow H$ be a nonlinear mapping, where $R$ is the set of real numbers. Then, Takahashi and Takahashi [21] considered the following generalized equilibrium problem:

$$
\text { Find } x \in C \text { such that } F(x, y)+\langle B x, y-x\rangle \geq 0, \quad \forall y \in C \text {. }
$$

The set of solutions of (1.1) is denoted by $G E P(F)$. If $B=0$, the generalized equilibrium problem (1.1) becomes the equilibrium problem for $F: C \times C \rightarrow R$, which is to find $x \in C$ such that

$$
F(x, y) \geq 0, \quad \forall y \in C .
$$

The first author was supported by the National Natural Science Foundation of China (grant number 10771228), the Science and Technology Research Project of Chinese Ministry of Education (grant number 206123), the Education Committee project Research Foundation of Chongqing (grant number KJ070816). The second author was partially supported by the grant NSC96-2628-E-110-014MY3.

(C) 2009 Australian Mathematical Society 0004-9727/2009 \$16.00 
The set of solutions of (1.2) is denoted by $E P(F)$.

The problem (1.1) is very general in the sense that it includes, as special cases, optimization problems, variational inequalities, minimax problems, the Nash equilibrium problem in noncooperative games and others; see, for instance, [5] and [1].

Recall that a mapping $S: C \rightarrow H$ is nonexpansive [7] if it holds that

$$
\|S x-S y\| \leq\|x-y\|, \quad \forall x, y \in C .
$$

We denote the set of fixed points of $S$ by $\operatorname{Fix}(S)$. It is known (see [7]) that $\operatorname{Fix}(S)$ is closed convex, but possibly empty. A mapping $A$ of $C$ into $H$ is called monotone if

$$
\langle A x-A y, x-y\rangle \geq 0
$$

for all $x, y \in C$. A mapping $A$ of $C$ into $H$ is called $\alpha$-inverse-strongly monotone if there exists a positive real number $\alpha$ such that

$$
\langle x-y, A x-A y\rangle \geq \alpha\|A x-A y\|^{2}
$$

for all $x, y \in C$. A mapping $A: C \rightarrow H$ is called $k$-Lipschitz-continuous if there exists a positive real number $k$ such that

$$
\|A x-A y\| \leq k\|x-y\|
$$

for all $x, y \in C$. It is easy to see that the class of $\alpha$-inverse-strongly-monotone mappings does not contain some important classes of mappings even in a finitedimensional case. For example, if the matrix in the corresponding linear complementarity problem is positively semidefinite, but not positively definite, then the mapping $A$ will be monotone and Lipschitz-continuous, but not $\alpha$-inverse-strongly monotone.

Let the mapping $A: C \rightarrow H$ be monotone and $k$-Lipschitz-continuous. The variational inequality problem is to find a $x \in C$ such that

$$
\langle A x, y-x\rangle \geq 0
$$

for all $y \in C$. The set of solutions of the variational inequality problem is denoted by $V I(C, A)$.

Takahashi and Takahashi [21] introduced the following iterative scheme for finding a common element of the set of solutions of problem (1.1) and the set of fixed points of a nonexpansive mapping in a Hilbert space. Starting with an arbitrary $u \in C$ and $x_{1} \in C$, define sequences $\left\{x_{n}\right\}$ and $\left\{u_{n}\right\}$ by

$$
\begin{cases}F\left(u_{n}, y\right)+\left\langle B x_{n}, y-u_{n}\right\rangle+\frac{1}{r_{n}}\left\langle y-u_{n}, u_{n}-x_{n}\right\rangle \geq 0, & \forall y \in C, \\ x_{n+1}=\beta_{n} x_{n}+\left(1-\beta_{n}\right) S\left[\alpha_{n} u+\left(1-\alpha_{n}\right) u_{n}\right], & \forall n \in N .\end{cases}
$$

They proved that under certain appropriate conditions imposed on $\left\{\alpha_{n}\right\},\left\{\beta_{n}\right\}$ and $\left\{r_{n}\right\}$, the sequence $\left\{x_{n}\right\}$ generated by (1.3) converges strongly to $z=P_{\operatorname{Fix}(S) \cap G E P(F)} u$. 
Some methods have been proposed to solve the problem (1.2); see, for instance, [5, 1, 4, 20, 18, 17, 12]. Recently, Combettes and Hirstoaga [4] introduced an iterative scheme of finding the best approximation to the initial data when $\operatorname{EP}(F)$ is nonempty and proved a strong convergence theorem. Takahashi and Takahashi [20] introduced the following iterative scheme by the viscosity approximation method for finding a common element of the set of solution (1.2) and the set of fixed points of a nonexpansive mapping in a Hilbert space. Starting with an arbitrary $x_{1} \in H$, define sequences $\left\{x_{n}\right\}$ and $\left\{u_{n}\right\}$ by

$$
\begin{cases}F\left(u_{n}, y\right)+\frac{1}{r_{n}}\left\langle y-u_{n}, u_{n}-x_{n}\right\rangle \geq 0, & \forall y \in C, \\ x_{n+1}=\alpha_{n} f\left(x_{n}\right)+\left(1-\alpha_{n}\right) S u_{n}, & \forall n \in N .\end{cases}
$$

They proved that under certain appropriate conditions imposed on $\left\{\alpha_{n}\right\}$ and $\left\{r_{n}\right\}$, the sequences $\left\{x_{n}\right\}$ and $\left\{u_{n}\right\}$ generated by (1.4) converge strongly to $z \in \operatorname{Fix}(S) \cap \operatorname{EP}(F)$, where $z=P_{\operatorname{Fix}(S) \cap E P(F)} f(z)$, where $f$ is a contraction on $H$. Tada and Takahashi [18] introduced some iterative schemes for finding a common element of the set of solutions of problem (1.2) and the set of fixed points of a nonexpansive mapping and proved a strong convergence theorem and a weak convergence theorem in a Hilbert space. Su et al. [17] introduced the following iterative scheme by the viscosity approximation method for finding a common element of the set of solutions of problem (1.2) and the set of fixed points of a nonexpansive mapping and the set of solutions of the variational inequality problem for an $\alpha$-inverse strongly monotone mapping in a Hilbert space. Starting with an arbitrary $x_{1} \in H$, define sequences $\left\{x_{n}\right\}$ and $\left\{u_{n}\right\}$ by

$$
\begin{cases}F\left(u_{n}, y\right)+\frac{1}{r_{n}}\left\langle y-u_{n}, u_{n}-x_{n}\right\rangle \geq 0, & \forall y \in C, \\ x_{n+1}=\alpha_{n} f\left(x_{n}\right)+\left(1-\alpha_{n}\right) S P_{C}\left(u_{n}-\lambda_{n} A u_{n}\right), & \forall n \in N .\end{cases}
$$

They proved that under certain appropriate conditions imposed on $\left\{\alpha_{n}\right\},\left\{r_{n}\right\}$ and $\left\{\lambda_{n}\right\}$, the sequences $\left\{x_{n}\right\}$ and $\left\{u_{n}\right\}$ generated by (1.5) converge strongly to $z \in \operatorname{Fix}(S) \cap$ $E P(F) \cap V I(C, A)$, where $z=P_{\text {Fix }(S) \cap E P(F) \cap V I(C, A)} f(z)$.

On the other hand, to solve the variational inequality problem in the finitedimensional Euclidean $R^{n}$, Korpelevich [9] introduced the following so-called extragradient method:

$$
\left\{\begin{array}{l}
x_{1}=x \in C, \\
y_{n}=P_{C}\left(x_{n}-\lambda A x_{n}\right), \\
x_{n+1}=P_{C}\left(x_{n}-\lambda A y_{n}\right),
\end{array}\right.
$$

for every $n=0,1,2, \ldots$, where $\lambda \in(0,1 / k)$. He showed that if $\operatorname{VI}(C, A)$ is nonempty, then the sequences $\left\{x_{n}\right\}$ and $\left\{y_{n}\right\}$, generated by (1.6), converge to the same point $z \in V I(C, A)$. The idea of the extragradient iterative process introduced by Korpelevich was successfully generalized and extended not only in Euclidean but also in Hilbert and Banach spaces; see, for example, the recent papers of He et al. [8], Gárciga et al. [6], Ceng and Yao [3], Solodov and Svaiter [16], and Solodov [15]. 
Moreover, Zeng and Yao [24] and Nadezhkina and Takahashi [10] introduced some iterative processes by an extragradient method for finding the common element of the set of fixed points of nonexpansive mappings and the set of solutions of variational inequality problem for a monotone, Lipschitz-continuous mapping. Yao and Yao [23] introduced an iterative process based on the extragradient method for finding the common element of the set of fixed points of nonexpansive mappings and the set of solutions of variational inequality problem for an $\alpha$-inverse strongly monotone mapping. Plubtieng and Punpaeng [12] introduced an iterative process based on the extragradient method for finding the common element of the set of fixed points of nonexpansive mappings, the set of solutions of an equilibrium problem and the set of solutions of the variational inequality problem for $\alpha$-inverse strongly monotone mappings.

In the present paper, by using the extragradient methods, we introduce an iterative process for finding the common element of the set of fixed points of a nonexpansive mapping, the set of solutions of a generalized equilibrium problem and the set of solutions of the variational inequality problem for a monotone, Lipschitz-continuous mapping in a Hilbert space. Then, we obtain a weak convergence theorem for three sequences generated by this process. Based on this result, we also obtain several interesting results. The results in this paper generalize and extend some well-known weak convergence theorems in the literature.

\section{Preliminaries}

Let $H$ be a real Hilbert space with inner product $\langle\cdot, \cdot\rangle$ and norm $\|\cdot\|$. Let $C$ be a nonempty closed convex subset of $H$. Let symbols $\rightarrow$ and $\rightarrow$ denote strong and weak convergence, respectively. In a real Hilbert space $H$, it is well known that

$$
\|\lambda x+(1-\lambda) y\|^{2}=\lambda\|x\|^{2}+(1-\lambda)\|y\|^{2}-\lambda(1-\lambda)\|x-y\|^{2}
$$

for all $x, y \in H$ and $\lambda \in[0,1]$.

For any $x \in H$, there exists a unique nearest point in $C$, denoted by $P_{C}(x)$, such that $\left\|x-P_{C}(x)\right\| \leq\|x-y\|$ for all $y \in C$. The mapping $P_{C}$ is called the metric projection of $H$ onto $C$. We know that $P_{C}$ is a nonexpansive mapping from $H$ onto $C$. It is also known that $P_{C} x \in C$ and

$$
\left\langle x-P_{C}(x), P_{C}(x)-y\right\rangle \geq 0
$$

for all $x \in H$ and $y \in C$.

It is easy to see that (2.1) is equivalent to

$$
\|x-y\|^{2} \geq\left\|x-P_{C}(x)\right\|^{2}+\left\|y-P_{C}(x)\right\|^{2}
$$

for all $x \in H$ and $y \in C$. Let $A$ be a monotone mapping of $C$ into $H$. In the context of the variational inequality problem the characterization of projection (2.1) implies the following:

$$
u \in V I(C, A) \Rightarrow u=P_{C}(u-\lambda A u), \quad \lambda>0,
$$


and

$$
u=P_{C}(u-\lambda A u) \text { for some } \lambda>0 \Rightarrow u \in V I(C, A) .
$$

It is also known that $H$ satisfies the Opial's condition [11], that is, for any sequence $\left\{x_{n}\right\} \subset H$ with $x_{n} \rightarrow x$, the inequality

$$
\liminf _{n \rightarrow \infty}\left\|x_{n}-x\right\|<\liminf _{n \rightarrow \infty}\left\|x_{n}-y\right\|
$$

holds for every $y \in H$ with $x \neq y$.

A set-valued mapping $T: H \rightarrow 2^{H}$ is called monotone if for all $x, y \in H, f \in T x$ and $g \in T y$ imply $\langle x-y, f-g\rangle \geq 0$. A monotone mapping $T: H \rightarrow 2^{H}$ is maximal if its graph $G(T)$ of $T$ is not properly contained in the graph of any other monotone mapping. It is known that a monotone mapping $T$ is maximal if and only if for $(x, f) \in H \times H,\langle x-y, f-g\rangle \geq 0$ for every $(y, g) \in G(T)$ implies $f \in T x$. Let $A$ be a monotone, $k$-Lipschitz-continuous mapping of $C$ into $H$ and let $N_{C} v$ be normal cone to $C$ at $v \in C$, that is, $N_{C} v=\{w \in H \mid\langle v-u, w\rangle \geq 0, \forall u \in C\}$. Define

$$
T v= \begin{cases}A v+N_{C} v & \text { if } v \in C, \\ \emptyset & \text { if } v \notin C .\end{cases}
$$

Then $T$ is maximal monotone and $0 \in T v$ if and only if $v \in V I(C, A)$ (see [13]).

For solving the equilibrium problem, let us assume that the bifunction $F$ satisfies the following conditions:

(A1) $F(x, x)=0$ for all $x \in C$;

(A2) $F$ is monotone, that is, $F(x, y)+F(y, x) \leq 0$ for any $x, y \in C$;

(A3) for each $x, y, z \in C$,

$$
\lim _{t \downarrow 0} F(t z+(1-t) x, y) \leq F(x, y) ;
$$

(A4) for each $x \in C, y \mapsto F(x, y)$ is convex and lower semicontinuous.

We recall some lemmas that are needed in the rest of this paper.

LEMMA 2.1 [1]. Let $C$ be a nonempty closed convex subset of $H$, let $F$ be a bifunction from $C \times C$ to $R$ satisfying conditions (A1)-(A4). Let $r>0$ and $x \in H$. Then, there exists $z \in C$ such that

$$
F(z, y)+\frac{1}{r}\langle y-z, z-x\rangle \geq 0, \quad \forall y \in C .
$$

LEMMA 2.2 [4]. Let $C$ be a nonempty closed convex subset of $H$, let $F$ be a bifunction from $C \times C$ to $R$ satisfying conditions (A1)-(A4). For $r>0$ and $x \in H$, define a mapping $T_{r}: H \rightarrow C$ as follows:

$$
T_{r}(x)=\left\{z \in C: F(z, y)+\frac{1}{r}\langle y-z, z-x\rangle \geq 0, \forall y \in C\right\}
$$

for all $x \in H$. Then, the following statements hold: 
(1) $T_{r}$ is single-valued;

(2) $T_{r}$ is firmly nonexpansive, that is, for any $x, y \in H$,

$$
\left\|T_{r}(x)-T_{r}(y)\right\|^{2} \leq\left\langle T_{r}(x)-T_{r}(y), x-y\right\rangle ;
$$

(3) $F\left(T_{r}\right)=E P(F)$;

(4) $E P(F)$ is closed and convex.

LEMMA 2.3 [14]. Let $H$ be a real Hilbert space, let $\left\{\alpha_{n}\right\}$ be a sequence of real numbers such that $0<a \leq \alpha_{n} \leq b<1$ for all $n=0,1,2, \ldots$, and let $\left\{v_{n}\right\}$ and $\left\{w_{n}\right\}$ be sequences in $H$ such that $\lim _{\sup _{n \rightarrow \infty}}\left\|v_{n}\right\| \leq c, \lim _{\sup _{n \rightarrow \infty}}\left\|w_{n}\right\| \leq c$, $\lim \sup _{n \rightarrow \infty}\left\|\alpha_{n} v_{n}+\left(1-\alpha_{n}\right) w_{n}\right\|=c$ for some $c \geq 0$. Then, $\lim _{n \rightarrow \infty}\left\|v_{n}-w_{n}\right\|$ $=0$.

LeMma 2.4 [22]. Let $H$ be a real Hilbert space, let $D$ be a nonempty closed convex subset of $H$. Let $\left\{x_{n}\right\}$ be a sequence in $H$. Suppose that, for all $u \in D$,

$$
\left\|x_{n+1}-u\right\| \leq\left\|x_{n}-u\right\|
$$

for every $n=0,1,2, \ldots$ Then, the sequence $\left\{P_{D} x_{n}\right\}$ converges strongly to some $z \in D$.

\section{A weak convergence theorem}

In this section, we show a weak convergence theorem which solves the problem of finding a common element of the set of solutions of a generalized equilibrium problem, the set of fixed points of a nonexpansive mapping and the set of the variational inequality for a monotone, Lipschitz-continuous mapping in a Hilbert space.

THEOREM 3.1. Let $C$ be a nonempty closed convex subset of a real Hilbert space $H$. Let $F$ be a bifunction from $C \times C$ to $R$ satisfying conditions $(A 1)-(A 4)$ and let $B$ be an $\alpha$-inverse-strongly monotone mapping of $C$ into $H$. Let $A$ be a monotone and $k$ Lipschitz-continuous mapping of $C$ into $H$. Let $S$ be a nonexpansive mapping of $C$ into $H$ such that $\Omega=\operatorname{Fix}(S) \cap \operatorname{VI}(C, A) \cap G E P(F) \neq \emptyset$. Let $\left\{x_{n}\right\},\left\{u_{n}\right\}$ and $\left\{y_{n}\right\}$ be sequences generated by

$$
\left\{\begin{array}{l}
x_{0}=x \in H \\
F\left(u_{n}, y\right)+\left\langle B x_{n}, y-u_{n}\right\rangle+\frac{1}{r_{n}}\left\langle y-u_{n}, u_{n}-x_{n}\right\rangle \geq 0, \quad \forall y \in C, \\
y_{n}=P_{C}\left(u_{n}-\lambda_{n} A u_{n}\right), \\
x_{n+1}=\alpha_{n} x_{n}+\left(1-\alpha_{n}\right) S P_{C}\left(u_{n}-\lambda_{n} A y_{n}\right),
\end{array}\right.
$$

for every $n=0,1,2, \ldots$ If $\left\{\lambda_{n}\right\} \subset[a, b]$ for some $a, b \in(0,1 / k),\left\{\alpha_{n}\right\} \subset[c, d]$ for some $c, d \in(0,1)$ and $\left\{r_{n}\right\} \subset[\gamma, \tau]$ for some $\gamma, \tau \in(0,2 \alpha)$. Then, $\left\{x_{n}\right\},\left\{u_{n}\right\}$ and $\left\{y_{n}\right\}$ converge weakly to $w \in \Omega$, where $w=\lim _{n \rightarrow \infty} P_{\Omega} x_{n}$. 
PROOF. Put $t_{n}=P_{C}\left(u_{n}-\lambda_{n} A y_{n}\right)$ for every $n=0,1,2, \ldots$ Let $u \in \Omega$ and let $\left\{T_{r_{n}}\right\}$ be a sequence of mappings defined as in Lemma 2.2. Then

$$
u=P_{C}\left(u-\lambda_{n} A u\right)=T_{r_{n}}\left(u-r_{n} B u\right) .
$$

From $u_{n}=T_{r_{n}}\left(x_{n}-r_{n} B x_{n}\right) \in C$ and the $\alpha$-inverse-strongly monotonicity of $B$, we have

$$
\begin{aligned}
\left\|u_{n}-u\right\|^{2} & =\left\|T_{r_{n}}\left(x_{n}-r_{n} B x_{n}\right)-T_{r_{n}}\left(u-r_{n} B u\right)\right\|^{2} \\
& \leq\left\|x_{n}-r_{n} B x_{n}-\left(u-r_{n} B u\right)\right\|^{2} \\
& \leq\left\|x_{n}-u\right\|^{2}-2 r_{n}\left\langle x_{n}-u, B x_{n}-B u\right\rangle+r_{n}^{2}\left\|B x_{n}-B u\right\|^{2} \\
& \leq\left\|x_{n}-u\right\|^{2}-2 r_{n} \alpha\left\|B x_{n}-B u\right\|^{2}+r_{n}^{2}\left\|B x_{n}-B u\right\|^{2} \\
& =\left\|x_{n}-u\right\|^{2}+r_{n}\left(r_{n}-2 \alpha\right)\left\|B x_{n}-B u\right\|^{2} \\
& \leq\left\|x_{n}-u\right\| .
\end{aligned}
$$

From (2.2), the monotonicity of $A$, and $u \in V I(C, A)$, we have

$$
\begin{aligned}
\left\|t_{n}-u\right\|^{2} \leq & \left\|u_{n}-\lambda{ }_{n} A y_{n}-u\right\|^{2}-\left\|u_{n}-\lambda_{n} A y_{n}-t_{n}\right\|^{2} \\
= & \left\|u_{n}-u\right\|^{2}-\left\|u_{n}-t_{n}\right\|^{2}+2 \lambda_{n}\left\langle A y_{n}, u-t_{n}\right\rangle \\
= & \left\|u_{n}-u\right\|^{2}-\left\|u_{n}-t_{n}\right\|^{2}+2 \lambda_{n}\left(\left\langle A y_{n}-A u, u-y_{n}\right\rangle\right. \\
& \left.+\left\langle A u, u-y_{n}\right\rangle+\left\langle A y_{n}, y_{n}-t_{n}\right\rangle\right) \\
\leq & \left\|u_{n}-u\right\|^{2}-\left\|u_{n}-t_{n}\right\|^{2}+2 \lambda_{n}\left\langle A y_{n}, y_{n}-t_{n}\right\rangle \\
\leq & \left\|u_{n}-u\right\|^{2}-\left\|u_{n}-y_{n}\right\|^{2}-2\left\langle u_{n}-y_{n}, y_{n}-t_{n}\right\rangle-\left\|y_{n}-t_{n}\right\|^{2} \\
& +2 \lambda_{n}\left\langle A y_{n}, y_{n}-t_{n}\right\rangle \\
= & \left\|u_{n}-u\right\|^{2}-\left\|u_{n}-y_{n}\right\|^{2}-\left\|y_{n}-t_{n}\right\|^{2} \\
& +2\left\langle u_{n}-\lambda_{n} A y_{n}-y_{n}, t_{n}-y_{n}\right\rangle .
\end{aligned}
$$

Further, since $y_{n}=P_{C}\left(u_{n}-\lambda_{n} A u_{n}\right)$ and $A$ is $k$-Lipschitz-continuous, we have

$$
\begin{aligned}
\left\langle u_{n}-\lambda_{n} A y_{n}-y_{n}, t_{n}-y_{n}\right\rangle= & \left\langle u_{n}-\lambda_{n} A u_{n}-y_{n}, t_{n}-y_{n}\right\rangle \\
& +\left\langle\lambda_{n} A u_{n}-\lambda_{n} A y_{n}, t_{n}-y_{n}\right\rangle \\
\leq & \left\langle\lambda_{n} A u_{n}-\lambda_{n} A y_{n}, t_{n}-y_{n}\right\rangle \\
\leq & \lambda_{n} k\left\|u_{n}-y_{n}\right\|\left\|t_{n}-y_{n}\right\| .
\end{aligned}
$$

So,

$$
\begin{aligned}
\left\|t_{n}-u\right\|^{2} \leq & \left\|u_{n}-u\right\|^{2}-\left\|u_{n}-y_{n}\right\|^{2}-\left\|y_{n}-t_{n}\right\|^{2}+2 \lambda_{n} k\left\|u_{n}-y_{n}\right\|\left\|t_{n}-y_{n}\right\| \\
\leq & \left\|u_{n}-u\right\|^{2}-\left\|u_{n}-y_{n}\right\|^{2}-\left\|y_{n}-t_{n}\right\|^{2} \\
& +\lambda_{n}{ }^{2} k^{2}\left\|u_{n}-y_{n}\right\|^{2}+\left\|t_{n}-y_{n}\right\|^{2} \\
= & \left\|u_{n}-u\right\|^{2}+\left(\lambda_{n}{ }^{2} k^{2}-1\right)\left\|u_{n}-y_{n}\right\|^{2} \\
\leq & \left\|u_{n}-u\right\|^{2}
\end{aligned}
$$


Therefore from (3.1) and (3.2), $x_{n+1}=\alpha_{n} x_{n}+\left(1-\alpha_{n}\right) S t_{n}$ and $u=S u$,

$$
\begin{aligned}
\left\|x_{n+1}-u\right\|^{2} & =\left\|\alpha_{n} x_{n}+\left(1-\alpha_{n}\right) S t_{n}-u\right\|^{2} \\
& \leq \alpha_{n}\left\|x_{n}-u\right\|^{2}+\left(1-\alpha_{n}\right)\left\|S t_{n}-u\right\|^{2} \\
& \leq \alpha_{n}\left\|x_{n}-u\right\|^{2}+\left(1-\alpha_{n}\right)\left\|t_{n}-u\right\|^{2} \\
& \leq \alpha_{n}\left\|x_{n}-u\right\|^{2}+\left(1-\alpha_{n}\right)\left[\left\|u_{n}-u\right\|^{2}+\left(\lambda_{n}{ }^{2} k^{2}-1\right)\left\|u_{n}-y_{n}\right\|^{2}\right] \\
& \leq\left\|x_{n}-u\right\|^{2}+\left(1-\alpha_{n}\right)\left(\lambda_{n}{ }^{2} k^{2}-1\right)\left\|u_{n}-y_{n}\right\|^{2} \\
& \leq\left\|x_{n}-u\right\|^{2}
\end{aligned}
$$

for every $n=0,1,2, \ldots$ Therefore, there exists $\theta=\lim _{n \rightarrow \infty}\left\|x_{n}-u\right\|$ and $\left\{x_{n}\right\}$ is bounded. From (3.1) and (3.2), we also obtain that $\left\{t_{n}\right\}$ and $\left\{u_{n}\right\}$ are bounded.

By (3.3), we have

$$
\left\|u_{n}-y_{n}\right\|^{2} \leq \frac{1}{\left(1-\alpha_{n}\right)\left(1-\lambda_{n}{ }^{2} k^{2}\right)}\left(\left\|x_{n}-u\right\|^{2}-\left\|x_{n+1}-u\right\|^{2}\right) .
$$

Hence, $\left\|u_{n}-y_{n}\right\| \rightarrow 0$.

By the same process as in (3.2), we also have

$$
\begin{aligned}
\left\|t_{n}-u\right\|^{2} & \leq\left\|u_{n}-u\right\|^{2}-\left\|u_{n}-y_{n}\right\|^{2}-\left\|y_{n}-t_{n}\right\|^{2}+2 \lambda_{n} k\left\|u_{n}-y_{n}\right\|\left\|t_{n}-y_{n}\right\| \\
& \leq\left\|u_{n}-u\right\|^{2}-\left\|u_{n}-y_{n}\right\|^{2}-\left\|y_{n}-t_{n}\right\|^{2}+\left\|u_{n}-y_{n}\right\|^{2}+\lambda_{n}{ }^{2} k^{2}\left\|t_{n}-y_{n}\right\|^{2} \\
& =\left\|u_{n}-u\right\|^{2}+\left(\lambda_{n}{ }^{2} k^{2}-1\right)\left\|y_{n}-t_{n}\right\|^{2} .
\end{aligned}
$$

Then, in contrast to (3.3),

$$
\begin{aligned}
\left\|x_{n+1}-u\right\|^{2} & \leq \alpha_{n}\left\|x_{n}-u\right\|^{2}+\left(1-\alpha_{n}\right)\left\|t_{n}-u\right\|^{2} \\
& \leq \alpha_{n}\left\|x_{n}-u\right\|^{2}+\left(1-\alpha_{n}\right)\left[\left\|u_{n}-u\right\|^{2}+\left(\lambda_{n}{ }^{2} k^{2}-1\right)\left\|y_{n}-t_{n}\right\|^{2}\right] \\
& \leq\left\|x_{n}-u\right\|^{2}+\left(1-\alpha_{n}\right)\left(\lambda_{n}{ }^{2} k^{2}-1\right)\left\|y_{n}-t_{n}\right\|^{2} \\
& \leq\left\|x_{n}-u\right\|^{2}
\end{aligned}
$$

and, rearranging as in (3.4),

$$
\left\|t_{n}-y_{n}\right\|^{2} \leq \frac{1}{\left(1-\alpha_{n}\right)\left(1-\lambda_{n}{ }^{2} k^{2}\right)}\left(\left\|x_{n}-u\right\|^{2}-\left\|x_{n+1}-u\right\|^{2}\right) .
$$

Hence, $\left\|t_{n}-y_{n}\right\| \rightarrow 0$. From $\left\|u_{n}-t_{n}\right\| \leq\left\|u_{n}-y_{n}\right\|+\left\|y_{n}-t_{n}\right\|$ we also have $\left\|u_{n}-t_{n}\right\| \rightarrow 0$. As $A$ is $k$-Lipschitz-continuous, we have $\left\|A y_{n}-A t_{n}\right\| \rightarrow 0$.

From (3.3) and (3.1), we have

$$
\begin{aligned}
\left\|x_{n+1}-u\right\|^{2} & \leq \alpha_{n}^{2}\left\|x_{n}-u\right\|^{2}+\left(1-\alpha_{n}\right)\left[\left\|u_{n}-u\right\|^{2}+\left(\lambda_{n}^{2} k^{2}-1\right)\left\|u_{n}-y_{n}\right\|^{2}\right] \\
& \leq \alpha_{n}^{2}\left\|x_{n}-u\right\|^{2}+\left(1-\alpha_{n}\right)\left\|u_{n}-u\right\|^{2} \\
& \leq \alpha_{n}^{2}\left\|x_{n}-u\right\|^{2}+\left(1-\alpha_{n}\right)\left[\left\|x_{n}-u\right\|^{2}+r_{n}\left(r_{n}-2 \alpha\right)\left\|B x_{n}-B u\right\|^{2}\right] \\
& =\left\|x_{n}-u\right\|^{2}+\left(1-\alpha_{n}\right) r_{n}\left(r_{n}-2 \alpha\right)\left\|B x_{n}-B u\right\|^{2} .
\end{aligned}
$$


It follows that

$$
\begin{aligned}
(1-d) \gamma(2 \alpha-\tau)\left\|B x_{n}-B u\right\|^{2} & \leq\left(1-\alpha_{n}\right) r_{n}\left(2 \alpha-r_{n}\right)\left\|B x_{n}-B u\right\|^{2} \\
& \leq\left\|x_{n}-u\right\|^{2}-\left\|x_{n+1}-u\right\|^{2} .
\end{aligned}
$$

Hence, we obtain $\left\|B x_{n}-B u\right\| \rightarrow 0$.

For $u \in \Omega$, from Lemma 2.2, we have

$$
\begin{aligned}
\left\|u_{n}-u\right\|^{2}= & \left\|T_{r_{n}}\left(x_{n}-r_{n} B x_{n}\right)-T_{r_{n}}\left(u-r_{n} B u\right)\right\|^{2} \\
\leq & \left\langle T_{r_{n}}\left(x_{n}-r_{n} B x_{n}\right)-T_{r_{n}}\left(u-r_{n} B u\right), x_{n}-r_{n} B x_{n}-\left(u-r_{n} B u\right)\right\rangle \\
= & \frac{1}{2}\left\{\left\|u_{n}-u\right\|^{2}+\left\|x_{n}-r_{n} B x_{n}-\left(u-r_{n} B u\right)\right\|^{2}-\| x_{n}-r_{n} B x_{n}\right. \\
& \left.-\left(u-r_{n} B u\right)-\left(u_{n}-u\right) \|^{2}\right\} \\
\leq & \frac{1}{2}\left\{\left\|u_{n}-u\right\|^{2}+\left\|x_{n}-u\right\|^{2}\right. \\
& \left.-\left\|x_{n}-r_{n} B x_{n}-\left(u-r_{n} B u\right)-\left(u_{n}-u\right)\right\|^{2}\right\} \\
= & \frac{1}{2}\left\{\left\|u_{n}-u\right\|^{2}+\left\|x_{n}-u\right\|^{2}-\left\|x_{n}-u_{n}\right\|^{2}\right. \\
& +2 r_{n}\left\langle B x_{n}-B u, x_{n}-u_{n}\right\rangle-r_{n}^{2}\left\|B x_{n}-B u\right\|^{2} .
\end{aligned}
$$

Hence,

$$
\begin{aligned}
\left\|u_{n}-u\right\|^{2} \leq & \left\|x_{n}-u\right\|^{2}-\left\|x_{n}-u_{n}\right\|^{2} \\
& +2 r_{n}\left\langle B x_{n}-B u, x_{n}-u_{n}\right\rangle-r_{n}^{2}\left\|B x_{n}-B u\right\|^{2} .
\end{aligned}
$$

Then, by (3.3) and (3.2),

$$
\begin{aligned}
\left\|x_{n+1}-u\right\|^{2} \leq & \alpha_{n}\left\|x_{n}-u\right\|^{2}+\left(1-\alpha_{n}\right)\left\|t_{n}-u\right\|^{2} \\
\leq & \alpha_{n}\left\|x_{n}-u\right\|^{2}+\left(1-\alpha_{n}\right)\left\|u_{n}-u\right\|^{2} \\
\leq & \alpha_{n}\left\|x_{n}-u\right\|^{2}+\left(1-\alpha_{n}\right)\left[\left\|x_{n}-u\right\|^{2}-\left\|x_{n}-u_{n}\right\|^{2}\right. \\
& \left.+2 r_{n}\left\langle B x_{n}-B u, x_{n}-u_{n}\right\rangle-r_{n}^{2}\left\|B x_{n}-B u\right\|^{2}\right] \\
\leq & \left\|x_{n}-u\right\|^{2}-\left(1-\alpha_{n}\right)\left\|x_{n}-u_{n}\right\|^{2} \\
& +\left(1-\alpha_{n}\right) 2 r_{n}\left\|B x_{n}-B u\right\|\left\|x_{n}-u_{n}\right\| .
\end{aligned}
$$

Hence,

$$
\begin{aligned}
(1-d)\left\|x_{n}-u_{n}\right\|^{2} \leq & \left(1-\alpha_{n}\right)\left\|x_{n}-u_{n}\right\|^{2} \\
\leq & \left\|x_{n}-u\right\|^{2}-\left\|x_{n+1}-u\right\|^{2} \\
& +\left(1-\alpha_{n}\right) 2 r_{n}\left\|B x_{n}-B u\right\|\left\|x_{n}-u_{n}\right\| .
\end{aligned}
$$

Since $\left\|B x_{n}-B u\right\| \rightarrow 0,\left\{x_{n}\right\}$ and $\left\{u_{n}\right\}$ are bounded, we obtain $\left\|x_{n}-u_{n}\right\| \rightarrow 0$. From $\left\|t_{n}-x_{n}\right\| \leq\left\|t_{n}-u_{n}\right\|+\left\|x_{n}-u_{n}\right\|$ we also have $\left\|t_{n}-x_{n}\right\| \rightarrow 0$.

For $u \in \Omega$, since $\left\|S t_{n}-u\right\| \leq\left\|t_{n}-u\right\| \leq\left\|x_{n}-u\right\|$, we have

$$
\limsup _{n \rightarrow \infty}\left\|S t_{n}-u\right\| \leq \theta \text {. }
$$


Furthermore,

$$
\lim _{n \rightarrow \infty}\left\|\alpha_{n}\left(x_{n}-u\right)+\left(1-\alpha_{n}\right)\left(S t_{n}-u\right)\right\|=\lim _{n \rightarrow \infty}\left\|x_{n+1}-u\right\|=\theta .
$$

By Lemma 2.3, we obtain $\lim _{n \rightarrow \infty}\left\|S t_{n}-x_{n}\right\|=0$.

Since

$$
\left\|S x_{n}-x_{n}\right\| \leq\left\|S x_{n}-S t_{n}\right\|+\left\|S t_{n}-x_{n}\right\| \leq\left\|x_{n}-t_{n}\right\|+\left\|S t_{n}-x_{n}\right\|,
$$

we have

$$
\left\|S x_{n}-x_{n}\right\| \rightarrow 0 .
$$

As $\left\{x_{n}\right\}$ is bounded, there exists a subsequence $\left\{x_{n_{i}}\right\}$ of $\left\{x_{n}\right\}$ such that $x_{n i} \rightarrow w$. From $\left\|x_{n}-u_{n}\right\| \rightarrow 0$, we obtain that $u_{n i} \rightarrow w$. Since $\left\{u_{n i}\right\} \subset C$ and $C$ is closed and convex, we obtain $w \in C$.

First, we show $w \in G E P(F)$. By $u_{n}=T_{r_{n}}\left(x_{n}-r_{n} B x_{n}\right)$, we know that

$$
F\left(u_{n}, y\right)+\left\langle B x_{n}, y-u_{n}\right\rangle+\frac{1}{r_{n}}\left\langle y-u_{n}, u_{n}-x_{n}\right\rangle \geq 0, \quad \forall y \in C .
$$

It follows from condition (A2) that

$$
\left\langle B x_{n}, y-u_{n}\right\rangle+\frac{1}{r_{n}}\left\langle y-u_{n}, u_{n}-x_{n}\right\rangle \geq F\left(y, u_{n}\right), \quad \forall y \in C .
$$

Hence,

$$
\left\langle B x_{n_{i}}, y-u_{n_{i}}\right\rangle+\left\langle y-u_{n_{i}}, \frac{u_{n_{i}}-x_{n_{i}}}{r_{n_{i}}}\right\rangle \geq F\left(y, u_{n_{i}}\right), \quad \forall y \in C .
$$

For $t$ with $0<t \leq 1$ and $y \in C$, let $y_{t}=t y+(1-t) w$. Since $y \in C$ and $w \in C$, we obtain $y_{t} \in C$. So, from (3.6) we have

$$
\begin{aligned}
\left\langle y_{t}-u_{n_{i}}, B y_{t}\right\rangle \geq & \left\langle y_{t}-u_{n_{i}}, B y_{t}\right\rangle-\left\langle y_{t}-u_{n_{i}}, B x_{n_{i}}\right\rangle \\
& -\left\langle y_{t}-u_{n_{i}}, \frac{u_{n_{i}}-x_{n_{i}}}{r_{n_{i}}}\right\rangle+F\left(y_{t}, u_{n_{i}}\right) \\
= & \left\langle y_{t}-u_{n_{i}}, B y_{t}-B u_{n_{i}}\right\rangle+\left\langle y_{t}-u_{n_{i}}, B u_{n_{i}}-B x_{n_{i}}\right\rangle \\
& -\left\langle y_{t}-u_{n_{i}}, \frac{u_{n_{i}}-x_{n_{i}}}{r_{n_{i}}}\right\rangle+F\left(y_{t}, u_{n_{i}}\right) .
\end{aligned}
$$

Since $\left\|u_{n_{i}}-x_{n_{i}}\right\| \rightarrow 0$, we have $\left\|B u_{n_{i}}-B x_{n_{i}}\right\| \rightarrow 0$. Further, from the inversestrongly monotonicity of $B$, we have $\left\langle y_{t}-u_{n_{i}}, B y_{t}-B u_{n_{i}}\right\rangle \geq 0$. So, from condition (A4) we have

$$
\left\langle y_{t}-w, B y_{t}\right\rangle \geq F\left(y_{t}, w\right)
$$


as $i \rightarrow \infty$. From conditions (A1) and (A4) and (3.7), we also have

$$
\begin{aligned}
0 & =F\left(y_{t}, y_{t}\right) \leq t F\left(y_{t}, y\right)+(1-t) F\left(y_{t}, w\right) \\
& \leq t F\left(y_{t}, y\right)+(1-t)\left\langle y_{t}-w, B y_{t}\right\rangle \\
& =t F\left(y_{t}, y\right)+(1-t) t\left\langle y-w, B y_{t}\right\rangle
\end{aligned}
$$

and, hence,

$$
0 \leq F\left(y_{t}, y\right)+(1-t)\left\langle y-w, B y_{t}\right\rangle .
$$

Letting $t \rightarrow 0$, we have, for each $y \in C$,

$$
F(w, y)+\langle y-w, B w\rangle \geq 0 .
$$

This implies that $w \in G E P(F)$.

We next show that $w \in \operatorname{Fix}(S)$. Assume $w \notin \operatorname{Fix}(S)$. Since $x_{n_{i}} \rightarrow w$ and $w \neq S w$, from the Opial theorem and (3.5) we have

$$
\begin{aligned}
\liminf _{i \rightarrow \infty}\left\|x_{n_{i}}-w\right\| & <\liminf _{i \rightarrow \infty}\left\|x_{n_{i}}-S w\right\| \\
& \leq \liminf _{i \rightarrow \infty}\left\{\left\|x_{n_{i}}-S x_{n_{i}}\right\|+\left\|S x_{n_{i}}-S w\right\|\right\} \\
& \leq \liminf _{i \rightarrow \infty}\left\|x_{n_{i}}-w\right\| .
\end{aligned}
$$

This is a contradiction. So, we obtain $w \in \operatorname{Fix}(S)$.

Finally, we show $w \in \operatorname{VI}(C, A)$. Let

$$
T v= \begin{cases}A v+N_{C} v & \text { if } v \in C, \\ \varnothing & \text { if } v \notin C .\end{cases}
$$

where $N_{C} v$ is the normal cone to $C$ at $v \in C$. We have already mentioned that in this case the mapping $T$ is maximal monotone, and $0 \in T v$ if and only if $v \in \operatorname{VI}(C, A)$. Let $(v, g) \in G(T)$. Then $T v=A v+N_{C} v$ and, hence, $g-A v \in N_{C} v$. So, we have $\langle v-t, g-A v\rangle \geq 0$ for all $t \in C$. On the other hand, from $t_{n}=P_{C}\left(u_{n}-\lambda_{n} A y_{n}\right)$ and $v \in C$ we have

$$
\left\langle u_{n}-\lambda_{n} A y_{n}-t_{n}, t_{n}-v\right\rangle \geq 0
$$

and, hence,

$$
\left\langle v-t_{n}, \frac{t_{n}-u_{n}}{\lambda_{n}}+A y_{n}\right\rangle \geq 0
$$

Therefore,

$$
\begin{aligned}
\left\langle v-t_{n_{i}}, g\right\rangle & \geq\left\langle v-t_{n_{i}}, A v\right\rangle \\
& \geq\left\langle v-t_{n_{i}}, A v\right\rangle-\left\langle v-t_{n_{i}}, \frac{t_{n_{i}}-u_{n_{i}}}{\lambda_{n_{i}}}+A y_{n_{i}}\right\rangle \\
& =\left\langle v-t_{n_{i}}, A v-A y_{n_{i}}-\frac{t_{n_{i}}-u_{n_{i}}}{\lambda_{n_{i}}}\right\rangle \\
& =\left\langle v-t_{n_{i}}, A v-A t_{n_{i}}+A t_{n_{i}}-A y_{n_{i}}-\frac{t_{n_{i}}-u_{n_{i}}}{\lambda_{n_{i}}}\right\rangle
\end{aligned}
$$




$$
\begin{aligned}
& =\left\langle v-t_{n_{i}}, A v-A t_{n_{i}}\right\rangle+\left\langle v-t_{n_{i}}, A t_{n_{i}}-A y_{n_{i}}\right\rangle-\left\langle v-t_{n_{i}}, \frac{t_{n_{i}}-u_{n_{i}}}{\lambda_{n_{i}}}\right\rangle \\
& \geq\left\langle v-t_{n_{i}}, A t_{n_{i}}-A y_{n_{i}}\right\rangle-\left\langle v-t_{n_{i}}, \frac{t_{n_{i}}-u_{n_{i}}}{\lambda_{n_{i}}}\right\rangle .
\end{aligned}
$$

Hence, we obtain $\langle v-w, g\rangle \geq 0$ as $i \rightarrow \infty$. Since $T$ is maximal monotone, we have $w \in T^{-1} 0$ and, hence, $w \in \operatorname{VI}(C, A)$. This implies $w \in \Omega$.

Let $\left\{x_{n_{j}}\right\}$ be another subsequence of $\left\{x_{n}\right\}$ such that $x_{n_{j}} \rightarrow z$. Then $z \in \Omega$. Let us show $w=z$. Assume that $w \neq z$. From the Opial condition,

$$
\begin{aligned}
\lim _{n \rightarrow \infty}\left\|x_{n}-w\right\| & =\liminf _{i \rightarrow \infty}\left\|x_{n_{i}}-w\right\|<\liminf _{i \rightarrow \infty}\left\|x_{n_{i}}-z\right\| \\
& =\lim _{n \rightarrow \infty}\left\|x_{n}-z\right\|=\liminf _{j \rightarrow \infty}\left\|x_{n_{j}}-z\right\| \\
& <\liminf _{j \rightarrow \infty}\left\|x_{n_{j}}-w\right\|=\lim _{n \rightarrow \infty}\left\|x_{n}-w\right\| .
\end{aligned}
$$

This is a contradiction. Thus, we have $w=z$. This implies that $x_{n} \rightarrow w \in \Omega$. Since $\left\|x_{n}-u_{n}\right\| \rightarrow 0$, we have $u_{n} \rightarrow w \in \Omega$. Since $\left\|y_{n}-u_{n}\right\| \rightarrow 0$, we also have $y_{n} \rightarrow w \in \Omega$.

Now put $w_{n}=P_{\Omega} x_{n}$. We show that $w=\lim _{n \rightarrow \infty} w_{n}$.

From $w_{n}=P_{\Omega} x_{n}$ and $w \in \Omega$, we have

$$
\left\langle w-w_{n}, w_{n}-x_{n}\right\rangle \geq 0 .
$$

From (3.3) and Lemma 2.4, we know that $\left\{w_{n}\right\}$ converges strongly to some $w_{0} \in \Omega$. Then

$$
\left\langle w-w_{0}, w_{0}-w\right\rangle \geq 0
$$

and, hence, $w=w_{0}$. The proof is now complete.

\section{Applications}

By Theorem 3.1, we can obtain the following interesting weak convergence theorems in a real Hilbert space.

THEOREM 4.1. Let $C$ be a nonempty closed convex subset of a real Hilbert space $H$. Let $F$ be a bifunction from $C \times C$ to $R$ satisfying conditions (A1)-(A4) and $B$ be an $\alpha$-inverse-strongly monotone mapping of $C$ into $H$. Let $S$ be a nonexpansive mapping of $C$ into $H$ such that $\operatorname{Fix}(S) \cap G E P(F) \neq \emptyset$. Let $\left\{x_{n}\right\}$ and $\left\{u_{n}\right\}$ be sequences generated by

$$
\left\{\begin{array}{l}
x_{0}=x \in H, \\
F\left(u_{n}, y\right)+\left\langle B x_{n}, y-u_{n}\right\rangle+\frac{1}{r_{n}}\left\langle y-u_{n}, u_{n}-x_{n}\right\rangle \geq 0, \quad \forall y \in C, \\
x_{n+1}=\alpha_{n} x_{n}+\left(1-\alpha_{n}\right) S u_{n},
\end{array}\right.
$$


for every $n=0,1,2, \ldots$ If $\left\{\alpha_{n}\right\} \subset[c, d]$ for some $c, d \in(0,1)$ and $\left\{r_{n}\right\} \subset[\gamma, \tau]$ for some $\gamma, \tau \in(0,2 \alpha)$. Then, $\left\{x_{n}\right\}$ and $\left\{u_{n}\right\}$ converge weakly to $w \in \operatorname{Fix}(S) \cap \operatorname{GEP}(F)$, where $w=\lim _{n \rightarrow \infty} P_{\mathrm{Fix}(S) \cap G E P(F)} x_{n}$.

Proof. Putting $A=0$, by Theorem 3.1 we obtain the desired result.

THEOREM 4.2 [18, Theorem 4.1]. Let $C$ be a nonempty closed convex subset of a real Hilbert space $H$. Let $F$ be a bifunction from $C \times C$ to $R$ satisfying conditions (A1)(A4). Let $S$ be a nonexpansive mapping of $C$ into $H$ such that $\operatorname{Fix}(S) \cap E P(F) \neq \emptyset$. Let $\left\{x_{n}\right\}$ and $\left\{u_{n}\right\}$ be sequences generated by

$$
\left\{\begin{array}{l}
x_{0}=x \in H, \\
F\left(u_{n}, y\right)+\frac{1}{r_{n}}\left\langle y-u_{n}, u_{n}-x_{n}\right\rangle \geq 0, \quad \forall y \in C, \\
x_{n+1}=\alpha_{n} x_{n}+\left(1-\alpha_{n}\right) S u_{n},
\end{array}\right.
$$

for every $n=0,1,2, \ldots$ If $\left\{\alpha_{n}\right\} \subset[c, d]$ for some $c, d \in(0,1)$ and $\left\{r_{n}\right\} \subset[\gamma,+\infty)$ for some $\gamma>0$. Then, $\left\{x_{n}\right\}$ and $\left\{u_{n}\right\}$ converge weakly to $w \in \operatorname{Fix}(S) \cap \operatorname{EP}(F)$, where $w=\lim _{n \rightarrow \infty} P_{\operatorname{Fix}(S) \cap E P(F)} x_{n}$.

Proof. Putting $A=0$ and $B=0$, by Theorem 3.1 we obtain the desired result.

Theorem 4.3 [10, Theorem 4.1]. Let $H$ be a real Hilbert space. Let $A$ be a monotone and $k$-Lipschitz-continuous mapping of $C$ into $H$. Let $S$ be a nonexpansive mapping of $H$ into itself such that $\operatorname{VI}(C, A) \cap \operatorname{Fix}(S) \neq \emptyset$. Let $\left\{x_{n}\right\}$ be a sequence generated by

$$
\left\{\begin{array}{l}
x_{0}=x \in H, \\
x_{n+1}=\alpha_{n} x_{n}+\left(1-\alpha_{n}\right) S\left(x_{n}-\lambda_{n} A\left(x_{n}-\lambda_{n} A x_{n}\right)\right),
\end{array}\right.
$$

for every $n=0,1,2, \ldots$ If $\left\{\lambda_{n}\right\} \subset[a, b]$ for some $a, b \in(0,1 / k),\left\{\alpha_{n}\right\} \subset[c, d]$ for some $c, d \in(0,1)$. Then, $\left\{x_{n}\right\}$ converges weakly to $w \in \operatorname{VI}(C, A) \cap \operatorname{Fix}(S)$, where $w=\lim _{n \rightarrow \infty} P_{V I(C, A) \cap \operatorname{Fix}(S)} x_{n}$.

Proof. Putting $C=H, F=0$ and $B=0$, then $P_{H}=I$, by Theorem 3.1 we obtain the desired result.

Let $P: H \rightarrow 2^{H}$ be a maximal monotone mapping. Then, for any $x \in H$ and $r>0$, consider $J_{r}^{P} x=\{z \in H \mid z+r B z \ni x\}$. Such $J_{r}^{P}$ is called the resolvent of $B$ and is denoted by $J_{r}^{P}=(I+r P)^{-1}$.

Theorem 4.4 [10, Theorem 4.2]. Let $H$ be a real Hilbert space. Let $A$ be a monotone and k-Lipschitz-continuous mapping of C into $H$. Let $P: H \rightarrow 2^{H}$ be a maximal monotone mapping such that $A^{-1}(0) \cap P^{-1}(0) \neq \emptyset$. Let $J_{r}^{P}$ be the resolvent of $P$ for each $r>0$. Let $\left\{x_{n}\right\}$ be a sequence generated by

$$
\left\{\begin{array}{l}
x_{0}=x \in H, \\
x_{n+1}=\alpha_{n} x_{n}+\left(1-\alpha_{n}\right) J_{r}^{P}\left(x_{n}-\lambda_{n} A\left(x_{n}-\lambda_{n} A x_{n}\right)\right),
\end{array}\right.
$$


for every $n=0,1,2, \ldots$ If $\left\{\lambda_{n}\right\} \subset[a, b]$ for some $a, b \in(0,1 / k),\left\{\alpha_{n}\right\} \subset[c, d]$ for some $c, d \in(0,1)$. Then, $\left\{x_{n}\right\}$ converges weakly to $w \in A^{-1}(0) \cap P^{-1}(0)$, where $w=\lim _{n \rightarrow \infty} P_{A^{-1}(0) \cap P^{-1}(0)} x_{n}$.

Proof. Putting $C=H, F=0$ and $B=0$, then we have $P_{H}=I, A^{-1}(0)=V I(C, H)$ and $F\left(J_{r}^{P}\right)=P^{-1}(0)$. We also know that $J_{r}^{P}$ is nonexpansive (see [19]); by Theorem 3.1 we obtain the desired result.

A mapping $T$ of a closed convex subset $C$ into itself is pseudocontractive if there holds that

$$
\langle T x-T y, x-y\rangle \leq\|x-y\|^{2}
$$

for all $x, y \in C$; see [2]. Obviously, the class of pseudocontractive mappings is more general than the class of nonexpansive mappings. Now we prove a weak convergence theorem of a new iterative process for finding a common element of the set of solutions of a generalized equilibrium problem, the set of fixed points of a nonexpansive mapping and the set of fixed points of a Lipschitz pseudocontractive mapping.

THEOREM 4.5. Let $C$ be a nonempty closed convex subset of a real Hilbert space $H$. Let $F$ be a bifunction from $C \times C$ to $R$ satisfying conditions (A1)-(A4) and let $B$ be an $\alpha$-inverse-strongly monotone mapping of $C$ into $H$. Let $T$ be a pseudocontrative and $m$-Lipschitz-continuous mapping of $C$ into itself. Let $S$ be a nonexpansive mapping of $C$ into itself such that $\operatorname{Fix}(S) \cap \operatorname{Fix}(T) \cap G E P(F) \neq \emptyset$. Let $\left\{x_{n}\right\},\left\{u_{n}\right\}$ and $\left\{y_{n}\right\}$ be sequences generated by

$$
\left\{\begin{array}{l}
x_{1}=x \in H, \\
F\left(u_{n}, y\right)+\left\langle B x_{n}, y-u_{n}\right\rangle+\frac{1}{r_{n}}\left\langle y-u_{n}, u_{n}-x_{n}\right\rangle \geq 0, \quad \forall y \in C, \\
y_{n}=u_{n}-\lambda_{n}\left(u_{n}-T u_{n}\right), \\
x_{n+1}=\alpha_{n} x_{n}+\left(1-\alpha_{n}\right) S P_{C}\left(u_{n}-\lambda_{n}\left(y_{n}-T y_{n}\right)\right),
\end{array}\right.
$$

for every $n=1,2, \ldots$ If $\left\{\lambda_{n}\right\} \subset[a, b]$ for some $a, b \in(0,1 /(m+1)),\left\{\alpha_{n}\right\} \subset$ $[c, d]$ for some $c, d \in(0,1)$ and $\left\{r_{n}\right\} \subset[\gamma, \tau]$ for some $\gamma, \tau \in(0,2 \alpha)$. Then, $\left\{x_{n}\right\},\left\{u_{n}\right\}$ and $\left\{y_{n}\right\}$ converge weakly to $w \in \operatorname{Fix}(S) \cap \operatorname{Fix}(T) \cap \operatorname{GEP}(F)$, where $w=\lim _{n \rightarrow \infty} P_{\operatorname{Fix}(S) \cap \operatorname{Fix}(T) \cap G E P(F)} x_{n}$.

Proof. Let $A=I-T$. Let us show the mapping $A$ is monotone and $(m+1)$ Lipschitz-continuous. From the definition of the mapping $A$, we have

$$
\begin{aligned}
\langle A x-A y, x-y\rangle & =\langle x-y-T x+T y, x-y\rangle \\
& =\|x-y\|^{2}-\langle T x-T y, x-y\rangle \\
& \geq\|x-y\|^{2}-\|x-y\|^{2}=0 .
\end{aligned}
$$


So, $A$ is monotone. We also have

$$
\begin{aligned}
\|A x-A y\|^{2} & =\|(I-T) x-(I-T) y\| \\
& =\|x-y\|^{2}+\|T x-T y\|^{2}-2\langle x-y, T x-T y\rangle \\
& \leq\|x-y\|^{2}+m\|x-y\|^{2}+2\|x-y\|\|T x-T y\| \\
& \leq\|x-y\|^{2}+m\|x-y\|^{2}+2 m\|x-y\|^{2}=(m+1)^{2}\|x-y\|^{2} .
\end{aligned}
$$

So, we have $\|A x-A y\| \leq(m+1)\|x-y\|$ and $A$ is $(m+1)$-Lipschitz-continuous. It is easy to check that $\operatorname{Fix}(T)=V I(C, A)$. By Theorem 3.1 we obtain the desired result.

THEOREM 4.6. Let $C$ be a nonempty closed convex subset of a real Hilbert space $H$. Let $T$ be a pseudocontractive and m-Lipschitz-continuous mapping of $C$ into itself. Let $P: H \rightarrow 2^{H}$ be a maximal monotone mapping such that $\operatorname{Fix}(T) \cap P^{-1}(0) \neq \emptyset$. Let $\left\{x_{n}\right\}$ and $\left\{y_{n}\right\}$ be sequences generated by

$$
\left\{\begin{array}{l}
x_{1}=x \in H \\
y_{n}=x_{n}-\lambda_{n}\left(x_{n}-T x_{n}\right) \\
x_{n+1}=\alpha_{n} x_{n}+\left(1-\alpha_{n}\right) J_{r}^{P}\left(x_{n}-\lambda_{n}\left(y_{n}-T y_{n}\right)\right)
\end{array}\right.
$$

for every $n=1,2, \ldots$ If $\left\{\lambda_{n}\right\} \subset[a, b]$ for some $a, b \in(0,1 /(m+1)),\left\{\alpha_{n}\right\} \subset[c, d]$ for some $c, d \in(0,1)$ and $\left\{r_{n}\right\} \subset[\gamma, \tau]$ for some $\gamma, \tau \in(0,2 \alpha)$. Then, $\left\{x_{n}\right\}$ and $\left\{y_{n}\right\}$ converge weakly to $w \in \operatorname{Fix}(T) \cap P^{-1}(0)$, where $w=\lim _{n \rightarrow \infty} P_{\operatorname{Fix}(T) \cap P^{-1}(0)} x_{n}$.

Proof. Let $C=H, F(x, y)=0$ for $x, y \in H$, we have $u_{n}=x_{n}$ for every $n=0$, $1,2, \ldots$ We also know that $J_{r}^{P}$ is nonexpansive, $F\left(J_{r}^{B}\right)=B^{-1}(0)$ and $P_{H}=I$, by Theorem 4.5 we obtain the desired result.

THEOREM 4.7. Let $C$ be a nonempty closed convex subset of a real Hilbert space $H$. Let $B$ be an $\alpha$-inverse-strongly monotone mapping of $C$ into $H$. Let $A$ be a monotone and $k$-Lipschitz-continuous mapping of $C$ into $H$. Let $S$ be a nonexpansive mapping of $C$ into $H$ such that $\operatorname{Fix}(S) \cap V I(C, A) \cap V I(C, B) \neq \emptyset$. Let $\left\{x_{n}\right\},\left\{u_{n}\right\}$ and $\left\{y_{n}\right\}$ be sequences generated by

$$
\left\{\begin{array}{l}
x_{0}=x \in H \\
u_{n}=P_{C}\left(x_{n}-r_{n} B x_{n}\right) \\
y_{n}=P_{C}\left(u_{n}-\lambda_{n} A u_{n}\right) \\
x_{n+1}=\alpha_{n} x_{n}+\left(1-\alpha_{n}\right) S P_{C}\left(u_{n}-\lambda_{n} A y_{n}\right)
\end{array}\right.
$$

for every $n=0,1,2, \ldots \quad$ If $\left\{\lambda_{n}\right\} \subset[a, b]$ for some $a, b \in(0,1 / k),\left\{\alpha_{n}\right\} \subset$ $[c, d]$ for some $c, d \in(0,1)$ and $\left\{r_{n}\right\} \subset[\gamma, \tau]$ for some $\gamma, \tau \in(0,2 \alpha)$. Then, $\left\{x_{n}\right\},\left\{u_{n}\right\}$ and $\left\{y_{n}\right\}$ converge weakly to $w \in \operatorname{Fix}(S) \cap \operatorname{VI}(C, A) \cap \operatorname{VI}(C, B)$, where $w=\lim _{n \rightarrow \infty} P_{\operatorname{Fix}(S) \cap V I(C, A) \cap V I(C, B)} x_{n}$. 
PROOF. In Theorem 3.1, put $F=0$. Then, we obtain that

$$
\left\langle B x_{n}, y-u_{n}\right\rangle+\frac{1}{r_{n}}\left\langle y-u_{n}, u_{n}-x_{n}\right\rangle \geq 0, \quad \forall y \in C, \forall n \in N .
$$

This implies that

$$
\left\langle y-u_{n}, u_{n}-\left(x_{n}-r_{n} B x_{n}\right)\right\rangle \geq 0, \quad \forall y \in C, \forall n \in N .
$$

So, we obtain that $u_{n}=P_{C}\left(x_{n}-r_{n} B x_{n}\right)$ for all $n \in N$. Then we obtain the desired result from Theorem 3.1.

\section{References}

[1] E. Blum and W. Oettli, 'From optimization and variational inequalities to equilibrium problems', Math. Stud. 63 (1994), 123-145.

[2] F. E. Browder and W. V. Petryshyn, 'Construction of fixed points of nonlinear mappings in Hilbert space', J. Math. Anal. Appl. 20 (1967), 197-228.

[3] L. C. Ceng and J. C. Yao, 'Viscosity relaxed-extragradient method for monotone variational inequalities and fixed point problems', J. Math. Inequalities 1 (2007), 225-241.

[4] P. L. Combettes and S. A. Hirstoaga, 'Equilibrium programming in Hilbert spaces', J. Nonlinear Convex Anal. 6 (2005), 117-136.

[5] S. D. Flam and A. S. Antipin, 'Equilibrium programming using proximal-like algorithms', Math. Program. 78 (1997), 29-41.

[6] R. Gárciga Otero and A. Iuzem, 'Proximal methods with penalization effects in Banach spaces', Numer. Funct. Anal. Optim. 25 (2004), 69-91.

[7] K. Goebel and W. A. Kirk, Topics on Metric Fixed-point Theory (Cambridge University Press, Cambridge, 1990).

[8] B.-S. He, Z.-H. Yang and X.-M. Yuan, 'An approximate proximal-extragradient type method for monotone variational inequalities', J. Math. Anal. Appl. 300 (2004), 362-374.

[9] G. M. Korpelevich, 'The extragradient method for finding saddle points and other problems', Matecon 12 (1976), 747-756.

[10] N. Nadezhkina and W. Takahashi, 'Weak convergence theorem by an extragradient method for nonexpansive mappings and monotone mappings', J. Optim. Theory Appl. 128 (2006), 191-201.

[11] Z. Opial, 'Weak convergence of the sequence of successive approximation for nonexpansive mappings', Bull. Amer. Math. Soc. 73 (1967), 561-597.

[12] S. Plubtieng and R. Punpaeng, 'A new iterative method for equilibrium problems and fixed point problems of nonexpansive mappings and monotone mappings', Appl. Math. Comput. 197 (2008), $548-558$.

[13] R. T. Rockafellar, 'On the maximality of sums of nonlinear monotone operators', Trans. Amer. Math. Soc. 149 (1970), 75-88.

[14] J. Schu, 'Weak and strong convergence to fixed points of asymptotically nonexpansive mappings', Bull. Austral. Math. Soc. 43 (1991), 153-159.

[15] M. V. Solodov, 'Convergence rate analysis of iteractive algorithms for solving variational inequality problem', Math. Program. 96 (2003), 513-528.

[16] M. V. Solodov and B. F. Svaiter, 'An inexact hybrid generalized proximal point algorithm and some new results on the theory of Bregman functions', Math. Oper. Res. 25 (2000), 214-230.

[17] Y. Su, M. Shang and X. Qin, 'An iterative method of solutions for equilibrium and optimization problems', Nonlinear Anal. (2007), doi:10.1016/j.na.2007.08.045

[18] A. Tada and W. Takahashi, 'Weak and strong convergence theorems for a nonexpansive mapping and an equilibrium problem', J. Optim. Theory Appl. 133 (2007), 359-370. 
[19] W. Takahashi, Nonlinear Functional Analysis (Yokohama Publishers, Yokohama, Japan, 2000).

[20] S. Takahashi and W. Takahashi, 'Viscosity approximation methods for equilibrium problems and fixed point problems in Hilbert spaces', J. Math. Anal. Appl. 331 (2006), 506-515.

[21] _ ' 'Strong convergence theorem for a generalized equilibrium problem and a nonexpansive mapping in a Hilbert space', Nonlinear Anal. (2008), doi:10.10.1016/j.na.2008.02.042

[22] W. Takahashi and M. Toyoda, 'Weak convergence theorems for nonexpansive mappings and monotone mappings', J. Optim. Theory Appl. 118 (2003), 417-428.

[23] Y. Yao and J.-C. Yao, 'On modified iterative method for nonexpansive mappings and monotone mappings', Appl. Math. Comput. 186(2) (2007), 1551-1558.

[24] L. C. Zeng and J. C. Yao, 'Strong convergence theorem by an extragradient method for fixed point problems and variational inequality problems', Taiwan. J. Math. 10 (2006), 1293-1303.

JIAN-WEN PENG, College of Mathematics and Computer Science, Chongqing Normal University, Chongqing 400047, People's Republic of China e-mail: jwpeng6@yahoo.com.cn

JEN-CHIH YAO, Department of Applied Mathematics, National Sun Yat-sen University Kaohsiung, Taiwan 804, R.O.C. 